\title{
LE DOSAGE RAPIDE DE LA MATIËRE GRASSE DU LAIT. GERBER OU BABCOCK?
}

\author{
Par M. Emm. POZZI-ESCOT \\ Chimiste I. C. N. \\ Professeur à l'Institut National Agronomique du Pérou \\ Membre de l'American Chemical Society et de la Society of Chemical Industry.
}

Pour beaucoup de chimistes, surtout en France et en Espagne et dans les pays où le français et l'espagnol sont les langues usitées, quand on parle des procédés d'analyse rapide de la matière grasse du lait, c'est le procédé de Gerber qu'on a en vue. Voilà vingt ans que j'enseigne la chimie analytique à de futurs ingénieurs agronomes et vingt ans que j'ai abandonné ce procédé, le seul que j'ai connu, comme tous les jeunes chimistes français, à mes débuts, le seul que j'ai trouvé dans mes auteurs classiques, de langue française et allemande, car à l'époque, la littérature chimique de langue allemande avait chez nous une influence considérable. Nous avons négligé sans raison la chimie de langue anglaise.

Ce sont les raisons qui m'ont conduit à abándonner, il y a déjà longtemps, le procédé de Gerber, pour le procédé américain de Babeock, qu'il me paraît convenable de mettre en évidence, aujourd'hui qu'une réaction se fait sentir et qu'on se préoceupe en France, des remarquables travaux de la chimie américaine, aujourd'hui que Le Lait a pris à tâche de nous faire connaître les méthodes qui sont à la base de l'analyse officielle des laits aux Etats-Unis.

Lorsque je pénétrai pour la première fois, en 1906, dans le laboratoire où je devais, par la suite, enseigner à tant de générations d'ingénieurs et faire en faveur de la science française tous les efforts que j'ai pu, les jeunes aspirants faisaient justement des exercices pratiques de laiterie sous la direction de mon prédécesseur. Je fus presque immédiatement témoin d'un douloureux accident, rare sans doute, mais l'impression fut pour moi définitive : le bouchon d'un tube de Gerber, mal fixé sans doute, sauta et l'imprudent ou le maladroit reçut en pleine figure le jet d'acide avec toutes les conséquences qu'on imagine facilement. Dès ce jour je me promis de ne jamais exposer mes élèves aux dangers de ce procédé.

Le Gerber est connu de tous, inutile d'insister sur sa technique. Qu'il me soit permis de dire cependant, ce qui n'est généralement pas su, que Gerber a fait connaître son procédé une quinzaine d'années après que Babcock eut publié tous les détails du sien; aussi ne s'étonnera-t-on pas de mon opinion : le Gerber n'est pas un perfectionnement au procédé original de Babcock. J'ai cherché en 
vain les raisons des complications introduites par Gerber au procédé original de Babcock : complieation des récipients, si simples, si dénués de tout danger avec le Babcock; complieation des réactifs, dont le mieux qu'on puisse dire a été dit par Lrndet : “qu'on ne peut expliquer scientifiquement leur avantage. ") Ce n'est du reste pas d'un seul coup que Gerber est arrivé au mode opératoire actuel ; beaucoup d'entre nous ont usé et useront, peut-être, de l'acide sulfurique acétique du début.

N'oublions pas que la différence essentielle du Gerber et du Babcoek, actuellement, hors l'appareillage, réside dans le fait que Gerber ajoute de l'aleool amylique au mélange de lait et d'acide. Quel est le rôle de cet alcool amylique? Il paraît qu'une fraction inconnue s'unirait à la matière grasse pour en facititer la séparation surtout de ses plus fines particules. C'est pour cette dernière raison qu'avec les laits écrémés, le Gerber permettrait une meilleure évaluation que le Babcock, dont la précision est dans ce cas très certainement fort en défaut, mais je suis tout à fait d'avis que, dans ce cas, les deux procédés se donnent bien la main.

Le principe utilisé par Babcock, dans son procédé d'analyse rapide du lait, est bien connu de tous, puisqu'il est appliqué dans le Gerber: 10 destruction de l'émulsion de caséine et de matière grasse formant le lait par l'action d'un acide sulfurique de force identique à celui usé par Gerber, et $2^{\circ}$ dissolution de la caséine dans l'excès d'acide, laquelle est favorisée par l'élévation de température. La matière grasse ainsi libérée à l'état fondu, e'est-à-dire liquide, est réunie par centrifugation dans une graduation où il est facile d'en évaluer le poids connaissant son volume et la densité correspondante à la température de l'observation. Mais Babcock, au lieu d'opérer dans un tube fermé hermétiquement, comme on le fait dans le Gerber, opère dans un petit flacon à long col, gradué et ouvert. L'introduction du lait et de l'acide se font facilement à l'aide de pipettes; le mélange parfait des deux est ensuite obtenu sans aucune crainte par un simple mouvement circulaire donné au ballon tenu par son col. Après une première centrifugation qui, dans le mélange chaud et très dense d'acide et de lait, sépare la matière grasse, on verse de l'eau bouillante dans le flaeon de manière à réunir toute la matière grasse pure dans le col gradué du ballon où une lecture facile donne la quantité cherchée. Pour faciliter la réunion de toute la matière grasse, une nouvelle centrifugation, même deux, sont nécessaires, ainsi qu'un passage au bain-marie, de manière à réaliser les lectures de niveau de matière grasse dans des conditions de dilatation bien fixes de celle-ci. Ces opérations se font dans des eirconstances mitieusement déterminées, même standardisées par le Gouvernement des Etats-Unis. Telles sont les grandes lignes du procédé, comme 
dans le eas du Gerber, un appareillage convenable simplifie toutes les opérations : mesures de lait, de réactifs, chauffage, centrifugations, lectures. Il n'y a pas lieu d'insister, e'est affaire de catalogue.

Qu'elle comparaison peut-on faire entre les deux procédés et pourquoi est-il préférable de prendre le Babeock ?

Deux raisons me semblent amplement suffisantes pour ralier au Babeock tous les suffrages, d'une manière générale, et sans discussion possible. D'abord, la simplicité, certainement plus grande du matériel opératoire, la parfaite connaissance des réactions chimiques et physiques auxquelles on a reeours. Le mélange des réactifs, absolument sans aueune projection possible, par suite sans danger; jamais d'opérations ratées par pertes au bouchon, impossibilité absolue de laisser des grumeaux de caséine dans la partie graduée, puisqu'en versant de l'acide on a soin de laver le col gradué par un léger mouvement circulaire et que le mélange de l'acide et du lait est tellement facile qu'il est impossible de le projeter dans la graduation; aucune néeessité de chercher les meilleures eonditions de lecture de la colonne de matière grasse, ou qu'elle se trouve dans le col gradué, la lecture en est immédiate.

En second lieu, on sait, et on a toujours su avec le Babcock, ce que l'on fait; il n'en a pas été de même, en général, avee le Gerber, et je n'en veux eomme preuve que les nombreux ouvrages, signés par les maîtres en ehimie du lait de l'heure présente; qure les nombreux bulletins d'analyse qui me sont tombés sous les yeux en vingt ans; que les très nombreuses lettres recues de mes collègues à la suite de quelques publications sur le sujet. Très généralement dans les pays où les données analytiques sur le lait ont été habituellement exprimées en grammes par litre, on a considéré que-le Gerber donnait le pour cent de matière grasse en grammes par volume, traduisant de cette manière les dires des manuels : le "Gerber donne la matière grasse en poids, quoique la leeture représente un volume de graisse. » Or, la graduation du Gerber' a été faite identique à celle du Babcock en grammes de matière grasse pour $100 \mathrm{gr}$. de lait, e'est-à-dire pour cent en poids de graisse pour poids de lait et non pour cent en poids de graisse pour volume de lait. Je ne connais pas d'ouvrages où ces faits soient clairement dits et je m'explique de la sorte les erreurs sans nombre qui se sont glissées dans une infinitê de travaux analytiques.

Avec le Gerber, il faut accepter les indications du matériel gradué du commerce les yeux fermés; il n'existe pas, que je sache, de données officielles eertaines pouvant servir à la vérification des graduations ou des dires du fabricant, d'où des erreurs très fréquentes et très notables entre le matériel fourni par différentes maisons et souvent même entre divers tubes et pipettes de la même maison; 
chacun peut s'en rendre compte avec facilité; le calibrage de la graduation des tubes se fera par pesée de mercure.

Babcock, chimiste de l'Université du Wisconsin, quand il a publié vers 1872 son procédé, en a fait une description des plus minitieuse, faisant connaître jusque dans leur moindre détail les données expérimentales servant à la graduation des divers tubes. Le souci de l'extrême précision a été tel qu'il a été tenu compte dans ces données des phénomènes physiques de viscosité du lait et de dilatation des graisses. On se rend compte, bien facilement, de l'importance de ces faits.

La viscosité du lait intervient dans la-mesure du volume de lait devant correspondre au poids de lait sur lequel se fait l'essai. C'est ainsi que les analyses se faisait sur 18 gr. de lait, ce poids est mesuré et les pipettes devant donner 18 gr. de lait sont d'une capacité en eau de 17 cc. 6 , car, vu la viscosité du lait, une fois pleine de ce liquide et abandonnée à l'écoulement libre, elles ne laissent écouler que $17 \mathrm{cc} .5$ de lait moyen normal. Une pipette à graduation eau ordinaire conduit done à une erreur. Comme, d'autre part, la matière grasse n'est pas pesée, mais évaluée en poids d'après son volume apparent à l'état liquide à chaud, la graduation fixe, pour l'évaluation de ce volume, est faite en tenant compte du coefficient de dilatation si considérable des graisses, pour une lecture à réaliser à $58^{\circ}$ exactement; pour la centrifugation, la vitesse que doit avoir la centrifuge est indiquée avec précision suivant son diamètre, de manière à assurer une séparation convenable ; le temps lui-même de la centrifugation nécessaire est indiqué à quelques secondes près. L'Association Officielle des Chimistes agricoles américains a publié les conditions rigoureusement standard de tout cet appareillage, dans des publications répandues à profusion et en général gratuites; la verrerie graduée servant à ces essais est donc d'un modèle absolument fixe, vérifiée avec une précision absolue, donnant au chimiste qui en fait usage une garantie maximum, comme nos poids poinçonnés. On s'explique alors que les chimistes qui usent de ce procédé, pour peu qu'ils se conforment aux instructions officielles, soient certains de trouver les mêmes résultats toujours et partout. Il y a là un magnifique exemple de l'importance de la codification dans les méthodes officielles d'analyse qui est à méditer profondément; ceux qui voudraient en suivre l'exemple doivent se pénétrer de la manière suivant laquelle on a pu arriver à ce résultat : ce n'est pas celle dont on use ehez nous.

Chacun peut vérifier son appareillage en cas de doute, car les données auxquelles il doit satisfaire sont officiellement publiées par le Gouvernement des U. S., bureau des Standards ; il suffit d'avoir une balance et des poids exacts pour être à même de faire un contrôle absolu. 
Quand j'ai voulu vérifier mes tubes de Gerber, je n'ai pu trouver d'indications certaines sur la base de cette graduation; la maison qui les fabrique n'a pu ou n'a pas voulu m'éclairer à cet égard; mes vérifications ont reposé sur des données personnelles; nul doute que cette absolue confiance à donner au fabricant, constitue un défaut de premier ordre.

Telles sont les raisons qui me paraissent amplement suffisantes, dans leur ensemble, pour justifier l'abandon complet du procédé de Gerber, tout au moins en France où nous avons la réputation d'aimer le simple et le concis.

\title{
PHÉNOMĖNES \\ DE DIFFUSION DES CONSTITUANTS DU LAIT DANS UN GEL DE GÉLOSE
}

\author{
( $2^{e}$ communigation) \\ Par le Dr Alb. J.-J. VANDEVELDE \\ Professeur à l'Institut agronomique et à l'Université de Gand.
}

Dans ma première communication (1), j'ai constaté qu'un gel de gélose n'est pas en état de s'opposer au passage de colloïdes comme les lactoprotéines, et que sans modifier l'état colloïdal du sol une partie des substances' solubles diffuse du sol vers le gel; les constituants du lait restent ainsi dans le même état dispersé que dans le lait lui-même. Après un certain temps, le gel de gélose contient les constituants solubles en équilibre de concentration avec le sol.

Dans cette communication, j'ai annoncé également que je projetais de mettre les sols de concentration diminuée au contact de nouveaux gels de gélose, de manière à diluer encore plus les constituants solubles, et à pouvoir établir, dans ce cas, l'état des protéines. J'ai fait cette recherche et j'ai aussi étudié l'influence exercée par du lactose et par du chlorure de sodium introduits dans le gel à diverses concentrations.

I. - J'ai essayé de reproduire sur du lait sans formol les phénomènes de diffusion afin de voir si le formol additionné exerçait une influence. J'ai fait usage, comme auparavant, de fioles plates de Soyka de $10 \mathrm{~cm}$. de diamètre ; dans chaque fiole j'ai introduit $50 \mathrm{cc}$. de gel de gélose à $2 \%$. Les fioles étaient réunies entre elles de manière à pouvoir faire passer, au moment voulu, aseptiquement le lait d'une fiole dans l'autre ; dans ce but, la première fiole porte un bouchon muni de deux tubes dont l'un est bouché d'ouate et destiné à exercer une pression dans la fiole, et l'autre parvient jusqu'au fond

(1) Le Lait, 1924, 4, p. 638-640. 